\title{
Oxidative stress regulates mitogen-activated protein kinases and c-Jun activation involved in heat stress and lipopolysaccharide-induced intestinal epithelial cell apoptosis
}

\author{
YANAN LIU ${ }^{1,2^{*}}$, ZHENGLIAN WANG $^{3 *}$, WEIDANG XIE ${ }^{4 *}$, ZHENGTAO GU $^{5}$, QIULIN XU ${ }^{2}$ and LEI SU ${ }^{2}$ \\ ${ }^{1}$ Department of Intensive Care Unit, Nanfang Hospital, Southern Medical University, Guangzhou, \\ Guangdong 510515; ${ }^{2}$ Department of ICU, Key Laboratory of Tropical Zone Trauma Care and Tissue Repair of \\ PLA, General Hospital of Guangzhou Military Command, Guangzhou, Guangdong 510010; ${ }^{3}$ Graduate School, \\ Guangzhou University of Chinese Medicine, Guangzhou, Guangdong 510120; ${ }^{4}$ The First Affiliated Hospital of \\ Guangdong Pharmaceutical University, Guangzhou, Guangdong 510000; ${ }^{5}$ Department of Intensive Care Unit, \\ The Third Affiliated Hospital of Southern Medical University, Guangzhou, Guangdong 510630, P.R. China
}

Received April 17,2016; Accepted March 23, 2017

DOI: $10.3892 / \mathrm{mmr} .2017 .6859$

\begin{abstract}
Heat stress and gut-derived endotoxinemia are common causes of multiple organ dysfunction syndrome in heat stroke patients. Evidence has demonstrated that cell apoptosis in the small intestine serves an important role in the pathogenesis of heatstroke, which leads to increased intestinal permeability to endotoxin or lipopolysaccharides (LPS) from the gut entering the circulation. However, little is known about the potential underlying mechanisms mediating heat stress combined with LPS-induced intestinal epithelial cell apoptosis. In the present study, LPS combined with heat stress induced production of reactive oxygen species (ROS), mitochondrial membrane potential disruption and cell apoptosis, which eventually led to increased intestinal permeability and reduced epithelial resistance in the IEC-6 cell line. Inductions in ROS, mitochondrial membrane potential disruption and cell apoptosis were detected by using an ROS assay kit, 5,5',6,6'-tetrachloro-1,1',3,3'tetraethylbenzimi dazo carbocyanine iodide dye kit and annexin V-fluorescein isothiocyanate apoptosis kit, respectively. The effect of ROS on mitogen activated protein kinases (MAPKs) and c-Jun activation was investigated using the antioxidant drug, butylated hydroxyanisole (BHA) by western blotting. The results of the
\end{abstract}

Correspondence to: Dr Qiulin Xu or Professor Lei Su, Department of ICU, Key Laboratory of Tropical Zone Trauma Care and Tissue Repair of PLA, General Hospital of Guangzhou Military Command, 111 Liu Hua Road, Guangzhou, Guangdong 510010, P.R. China

E-mail: 123302688@qq.com

E-mail: slei_ICU@163.com

*Contributed equally

Key words: heat stress, apoptosis, reactive oxygen species, mitogen activated protein kinase, c-Jun, IEC-6 cells present study demonstrated that ROS is essential to activate p38, extracellular signal-regulated kinase (ERK) and c-Jun, but not c-Jun N-terminal kinase (JNK), in LPS combined with heat stress treated cells. Furthermore, ROS, and activation of p38, JNK and c-Jun, were revealed to serve pro-apoptosis roles which aggravated damage to epithelial barrier integrity, as assessed by flow cytometry using Annexin V-fluorescein isothiocyanate staining and pretreatment of cells with specific inhibitors of ROS, JNK, p38 and c-Jun (BHA, SP600125, SB203580 and c-Jun peptide, respectively). Transepithelial electrical resistance and horseradish peroxidase permeability were detected in cells treated with LPS combined with heat stress, which revealed that ERK serves an anti-apoptosis role, as determined by pretreatment of cells with PD98059, a specific inhibitor of ERK. In conclusion, these findings suggested a novel role of the ROS signaling pathway which involved activation of MAPKs and c-Jun, following LPS combined with heat stress-induced IEC- 6 cell apoptosis and impairment of the epithelial barrier. These results may facilitate understanding of pathological conditions involving ROS, such as heat stroke.

\section{Introduction}

Heat stress is a common stressful factor that affects many biological systems (1). In the process of heat stroke, intestinal epithelial cells are attacked by environmental heat and stimulated by intestinal bacteria and bacterial lipopolysaccharide (LPS) (2). Heat stress-induced intestinal epithelial cell injury and apoptosis contributes to intestinal hyperpermeability. Furthermore, bacterial products from the intestinal lumen entering into the circulatory system causes systemic inflammatory response syndrome and multiple organ failure (3-5). In our previous study, heat stress was demonstrated to induce apoptosis via transcription-independent p53-mediated mitochondrial signaling pathways in human umbilical vein endothelial cells (6). LPS is a major cell wall component in gram-negative bacteria that has been demonstrated to induce 
apoptosis and injury in various cell types (7). However, little is known about the biological effects of heat stress combined with LPS on intestinal epithelial cell apoptosis.

Reactive oxygen species (ROS) components, including superoxide anions, hydrogen peroxide and hydroxyl radicals, are typically generated in the mitochondria and serve as signaling intermediates $(8,9)$. Under physiological conditions, generated ROS are rapidly eliminated by antioxidant enzymes, including superoxide dismutases, catalase, glutathione peroxidases and peroxiredoxins (8). Numerous studies link oxidative stress with heat stress or LPS, and suggest synergistic augmentation of cell death and increased ROS generation in certain cells $(10,11)$.

The mitogen activated protein kinase (MAPK) cascades are activated by various cellular stresses and growth factors. Extracellular signal-regulated kinase (ERK), c-Jun NH2-terminal kinase (JNK) and p38 are members of well-characterized subfamilies of MAPK, and these enzymes have been implicated in the increased sensitivity to heat stress-induced cell apoptosis exhibited by IEC-6 cells (12-14). c-Jun is the most extensively studied protein of activator protein-1 components and has also been linked to apoptosis. The phosphorylation of c-Jun at Ser 63 and 73 is known to increase c-Jun activity (15-18). However, it remains unknown whether the MAPKs and c-Jun activation serves a critical role in LPS combined with heat stress-induced apoptosis, or if ROS has effects on MAPK and c-Jun signaling pathways under these conditions.

The present study aimed to investigate whether generation of reactive oxygen species (ROS) is a critical mediator in LPS combined with heat stress-induced apoptosis, and whether this may involve MAPK and c-Jun activation. Furthermore, whether cell apoptosis and permeability is exacerbated by inhibition of ERK1/2 (PD98059), JNK (SP600125), p38 (SB203580) and c-Jun (c-Jun peptide) activation was investigated.

\section{Materials and methods}

Cell culture and treatments. IEC-6 cells and Caco-2 cells were purchased from Shanghai Institute of Cell Biology, Chinese Academy of Sciences (Shanghai, China). Cells were cultured in Dulbecco's modified Eagle's medium (DMEM) supplemented with $10 \%$ (v/v) fetal bovine serum (FBS), $100 \mathrm{U} / \mathrm{ml}$ penicillin and $100 \mu \mathrm{g} / \mathrm{ml}$ streptomycin (Invitrogen; Thermo Fisher Scientific, Inc., Waltham, MA, USA) at $37^{\circ} \mathrm{C}$ in a humidified atmosphere of $5 \% \mathrm{CO}_{2}$ and $95 \%$ air. Culture dishes were placed into a circulating water bath at $37 \pm 0.5^{\circ} \mathrm{C}$ for control, or at $42 \pm 0.5^{\circ} \mathrm{C}$ for heat stress for $60 \mathrm{~min}$. The culture media was replaced with fresh media and the cells were further incubated at $37^{\circ} \mathrm{C}$ for $6 \mathrm{~h}$.

Intracellular ROS level. Levels of intracellular ROS were assessed using a ROS assay kit (Beyotime Institute of Biotechnology, Haimen, China). Dichlorofluorescein diacetate (DCFH-DA; Molecular Probes; Thermo Fisher Scientific, Inc.) enters the cells and reacts with ROS, producing the fluorophore DCF. Briefly, cells were exposed to 1,5 or $10 \mu \mathrm{g} / \mathrm{ml}$ LPS for $30 \mathrm{~min}$ at $37^{\circ} \mathrm{C}$, exposed to $1 \mu \mathrm{g} / \mathrm{ml}$ LPS for $5,15,30,60$ or $90 \mathrm{~min}$, or cells were treated with $1 \mu \mathrm{g} / \mathrm{ml}$ LPS combined with heat stress $\left(42^{\circ} \mathrm{C}\right)$ simultaneously for $60 \mathrm{~min}$, the cell culture media for each plate was then replaced with fresh media and the cells were further incubated at $37^{\circ} \mathrm{C}$ for $6 \mathrm{~h}$, control cells were always incubated at $37^{\circ} \mathrm{C}$. Control cells were treated with PBS instead of LPS. Cells $\left(3 \times 10^{5}\right)$ were harvested, washed with serum-free DMEM culture medium, and stained with $10 \mu \mathrm{M}$ DCFH-DA for $30 \mathrm{~min}$ at $37^{\circ} \mathrm{C}$ in the dark. Following this, cells were harvested, washed and resuspended in serum-free DMEM culture medium three times. The fluorescence intensity was determined using a flow cytometer (FACSCanto $^{\mathrm{TM}}$ II; BD Biosciences, San Jose, CA, USA) and analyzed using FlowJo software version 9.0 (Tree Star, Inc., Ashland, OR, USA).

Mitochondrial membrane potential assay. The fluorescent dye 5,5',6,6'-tetrachloro-1,1',3,3'tetraethylbenzimidazo carbocyanine iodide (JC-1; Molecular Probes; Thermo Fisher Scientific, Inc.) was used to detect the mitochondrial membrane potential. IEC- 6 cells were treated with LPS $(1 \mu \mathrm{g} / \mathrm{ml})$, heat stress $\left(42^{\circ} \mathrm{C}\right.$ for $\left.60 \mathrm{~min}\right)$, or a combination of LPS and heat stress, and further incubated for $12 \mathrm{~h}$ at $37^{\circ} \mathrm{C}$. Cells were washed three times with PBS. A JC-1 kit was used to detect the mitochondrial cross membrane potential. Results were observed through a fluorescence microscope.

Flow cytometric analysis of cell apoptosis using Annexin $V$-fluorescein isothiocyanate (FITC)/propidium iodide (PI) staining. Cells were pretreated with butylated hydroxyanisole (BHA; $5 \mu \mathrm{M}$; Abcam, Cambridge, MA, USA), SP600125 (10 $\mu \mathrm{M}$; Cell Signaling Technology, Inc., Danvers, MA, USA), SB203580 (5 $\mu \mathrm{M}$; Cell Signaling Technology, Inc.), PD98059 (10 $\mu \mathrm{M}$; Cell Signaling Technology, Inc.) and c-Jun peptide (10 $\mu \mathrm{M}$; R\&D Systems, Inc., Minneapolis, MN, USA), the specific inhibitors of ROS, JNK, p38, ERK and c-Jun, respectively, at $37^{\circ} \mathrm{C}$ for $30 \mathrm{~min}$ prior to simultaneous treatment with $1 \mu \mathrm{g} / \mathrm{ml} \mathrm{LPS}$ and heat stress $\left(42^{\circ} \mathrm{C}\right)$ for $60 \mathrm{~min}$, followed by a recovery period at $37^{\circ} \mathrm{C}$ for $6 \mathrm{~h}$. Control cells were treated with PBS instead of LPS and were always incubated at $37^{\circ} \mathrm{C}$. Cell apoptosis was analyzed using an Annexin V-FITC apoptosis kit (Lianke Biological Engineering Co., Ltd., Zhejiang, China) using a flow cytometer according to the manufacturer's protocol. Cells $\left(1 \times 10^{6}\right)$ cells were collected, washed in ice-cold PBS, and resuspended in the binding buffer containing $5 \mu 1$ FITC Annexin V and $10 \mu 1$ PI stain. The suspension was mixed and incubated at room temperature for $10 \mathrm{~min}$. Samples were subsequently analyzed using a flow cytometer.

Measurement of transepithelial electrical resistance (TEER). Approximately $2 \times 10^{6}$ IEC- 6 and Caco- 2 cells per well were seeded into collagen-coated Transwell membrane inserts separately $(6.5$-mm diameter inserts; 3 -mm pore size; Corning Incorporated, Corning, NY, USA) with $200 \mathrm{ml}$ culture medium added to the apical chamber and $600 \mathrm{ml}$ to the basolateral chamber. Cells were treated with LPS $(1 \mu \mathrm{g} / \mathrm{ml})$ for $60 \mathrm{~min}$ at $37^{\circ} \mathrm{C}$, heat stress $\left(42^{\circ} \mathrm{C}\right.$ for $\left.60 \mathrm{~min}\right)$ or simultaneous treatment with a combination of LPS $(1 \mu \mathrm{g} / \mathrm{ml})$ and heat stress $\left(42^{\circ} \mathrm{C}\right)$ for $60 \mathrm{~min}$. the cells were subsequently returned to a temperature of $37^{\circ} \mathrm{C}$, after $0,2,6$ and $12 \mathrm{~h}$ recovery period, the electrical resistance of confluent polarized IEC- 6 and Caco-2 monolayers was measured by TEER with an electrical resistance system (EVOM; World Precision Instruments GmbH, Berlin, 
Germany) at 0, 2, 6 and $12 \mathrm{~h}$, separately. A pair of electrodes were placed at each of the apical and basolateral chambers of three different points to evaluate TEER.

Intestinal paracellular permeability assay. Intestinal paracellular permeability across cell monolayers was determined by measuring the flux of horseradish peroxidase (HRP; type V; Sigma-Aldrich; Merck KGaA, Darmstadt, Germany). $\operatorname{HRP}\left(3.4 \times 10^{-6} \mathrm{~mol} / \mathrm{l}\right)$ was added to the medium in the apical chamber of Transwell chambers. After pre-treatment with LPS for $30 \mathrm{~min}$ and exposure to heat stress for $1 \mathrm{~h}$, samples were carefully taken from basolateral chambers and assayed for HRP by TMB HRP Color Development Solution for ELISA (Beyotime, China) 6 h later. Enzyme activity was determined from the rate of increase in optical density at a wavelength of $370 \mathrm{~nm}$ by an automatic microplate reader (SpectraMax ${ }^{\circledR}$ M5; Molecular Devices, LLC, Sunnyvale, CA, USA).

Western blot analysis. Protein concentration was determined by western blotting. IEC- 6 cells were exposed to LPS $(1 \mu \mathrm{g} / \mathrm{ml})$ for $60 \mathrm{~min}$ at $37^{\circ} \mathrm{C}$, heat stress $\left(42^{\circ} \mathrm{C}\right.$ for $\left.60 \mathrm{~min}\right)$ or simultaneous treatment with a combination of LPS $(1 \mu \mathrm{g} / \mathrm{ml})$ and heat stress treatment $\left(42^{\circ} \mathrm{C}\right)$ for $60 \mathrm{~min}$, and the cells were returned to a temperature of $37^{\circ} \mathrm{C}$ for $6 \mathrm{~h}$. In addition, cells were pretreated with or without BHA for $30 \mathrm{~min}$ at $37^{\circ} \mathrm{C}$, which was followed by simultaneous treatment with LPS $(1 \mu \mathrm{g} / \mathrm{ml})$ and heat stress $\left(42^{\circ} \mathrm{C}\right)$ with incubation for 0 , 15, 30 or $60 \mathrm{~min}$. Control cells treated with PBS instead of LPS and were incubated at $37^{\circ} \mathrm{C}$. Cells were homogenized in radioimmunoprecipitation assay lysis buffer with phenylmethylsulfonyl fluoride (Sigma-Aldrich; Merck KGaA). Following centrifugation at $14,000 \mathrm{x}$ at $4^{\circ} \mathrm{C}$ for $10 \mathrm{~min}$, the supernatants were used for western blot analysis. Protein concentration was determined by a Bicinchoninic Acid Protein Assay kit (Thermo Fisher Scientific, Inc.). Proteins (20 $\mu \mathrm{g} /$ well) were separated by SDS-PAGE using $10 \%$ SDS polyacrylamide gels and transferred onto polyvinylidene difluoride membranes. Membranes were blocked with blocking solution (5\% skimmed milk diluted with PBS) at room temperature for $2 \mathrm{~h}$, followed by incubation with primary antibodies. The following rabbit primary antibodies were used at a 1:2,000 dilution: GAPDH (cat. no. ab70699; Abcam), phosphorylated (p)-JNK (cat. no. 4668; Cell Signaling Technology, Inc.), p-p38 (cat. no. 4511; Cell Signaling Technology, Inc.), p-ERK1/2 (cat. no. 4370; Cell Signaling Technology, Inc.), JNK (cat. no. 9252; Cell Signaling Technology, Inc.), p38 (cat.no. 8690; Cell Signaling Technology, Inc.), ERK1/2 (cat. no. 4695; Cell Signaling Technology, Inc.), c-Jun (cat. no. 9165p; Cell Signaling Technology, Inc.), p-c-Jun (cat. no. 8222S; Cell Signaling Technology, Inc., Danvers, MA, USA), caspase-3 (cat. no. 14220S; Cell Signaling Technology, Inc.) and cleaved caspase-3 (cat. no. 9654S; Cell Signaling Technology, Inc.) overnight at $4^{\circ} \mathrm{C}$. A HRP-conjugated goat anti-rabbit IgG antibody served as the secondary antibody (1:5,000; cat. no. 7074; Cell Signaling Technology, Inc.) for incubation at room temperature for $2 \mathrm{~h}$. Proteins were visualized using an Enhanced Chemiluminescence reagent (Pierce; Thermo Fisher Scientific, Inc.). Membranes were exposed to light-sensitive film and quantified using Image $\mathbf{J}$ software version 1.3.4.67 (National Institutes of Health, Bethesda, MD, USA).
Statistical analysis. All data were analyzed for statistical significance using SPSS software version 13.0 (SPSS, Chicago, IL, USA). Data are expressed as the mean \pm standard deviation from at least three independent experiments performed in duplicate. One-way analysis of variance was performed followed by Fisher's least significant difference post hoc test for multiple comparisons. ${ }^{*} \mathrm{P}<0.05$ was considered to indicate a statistically significant difference.

\section{Results}

Heat stress plus LPS increases the production of ROS and permeability in IEC-6 cells. Based on the evidence that ROS generation serves as an important role in heat stress and LPS, the present study examined whether heat stress associated with LPS influences ROS accumulation in IEC-6 cells. Cells were exposed to 1,5 or $10 \mu \mathrm{g} / \mathrm{ml}$ of LPS for $30 \mathrm{~min}$. Following incubation with LPS, levels of intracellular ROS increased in a dose-dependent manner (Fig. 1A). As presented in Fig. 1B, a time-course experiment revealed that $1 \mu \mathrm{g} / \mathrm{ml}$ LPS increased intracellular ROS levels in IEC-6 cells compared with controls, with maximal induction being observed after a $15-\mathrm{min}$ incubation and returning to baseline levels at 90 min, $\mathrm{H}_{2} \mathrm{O}_{2}$ was used as a positive control. To test the combined effects of LPS treatment with heat stress on ROS accumulation, IEC-6 were treated with $1 \mu \mathrm{g} / \mathrm{ml}$ LPS and exposed to heat stress $\left(42^{\circ} \mathrm{C}\right)$ for $60 \mathrm{~min}$. At $6 \mathrm{~h}$ after treatment, the levels of intracellular ROS in IEC- 6 cells exposed to both LPS and heat stress were increased compared with controls and cells exposed to heat stress alone (Fig. 1C).

Heat stress and LPS lead to the mitochondrial membrane potential disruption in IEC-6 cells. Mitochondria may provide a permanent source of ROS under physiological conditions. Alterations in the mitochondrial membrane potential $(\Delta \Psi \mathrm{m})$ were assessed using JC-1. When mitochondrial membrane potential is high, JC1 accumulates in the mitochondrial matrix, forming JC-1 polymer aggregates which produces red fluorescence. When the mitochondrial membrane potential is lower, JC1 cannot accumulate in the mitochondrial matrix, JC-1 remains a monomer, which produces green fluorescence. As presented in Fig. 2, LPS and heat stress resulted in the reduction of mitochondrial membrane potential, particularly when used in combination.

Effect of heat stress and LPS on intestinal epithelial cell apoptosis and barrier function. There were significant differences in early apoptotic rates among the control, LPS, heat stress, and LPS combined with heat stress groups. Early apoptotic rates in the LPS, heat stress, and LPS combined with HS groups were significantly higher compared with the control group. Early apoptotic rates in the heat stress combined with LPS group were also increased compared with the LPS and heat stress groups (Fig. 3A). These results indicated that heat stress or LPS may increase apoptosis of IEC- 6 cells, and that the combination of these two treatments may produce a synergistic effect.

The present study examined the effect of LPS and heat stress on the effects of heat stress-induced dysfunction of the intestinal epithelial barrier in IEC-6 and Caco-2 monolayers. 

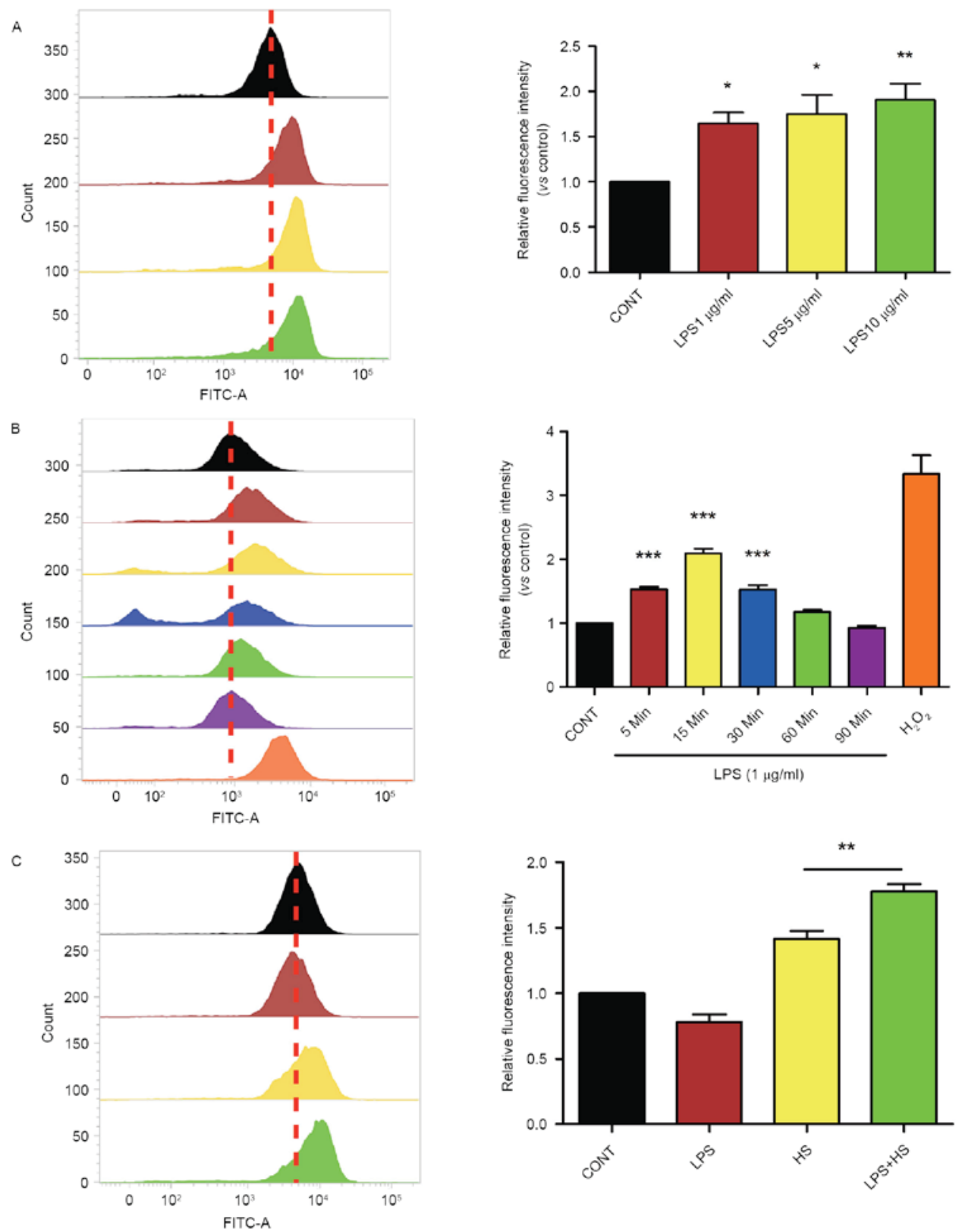

Figure 1. Effect of heat stress and LPS on ROS production in IEC-6 cells. (A) Cells were incubated with LPS (1-10 $\mu \mathrm{g} / \mathrm{ml})$ for $30 \mathrm{~min}$. (B) Cells incubated with LPS $(1 \mu \mathrm{g} / \mathrm{ml})$ for the indicated times. (C) Cells incubated with LPS $(1 \mu \mathrm{g} / \mathrm{ml})$ and subsequently exposed to heat stress $\left(42^{\circ} \mathrm{C}\right)$ for 60 min or not. ROS levels were assessed by dichlorofluorescein diacetate staining. Data are presented as the mean \pm standard deviation of three independent experiments, ${ }^{*} \mathrm{P}<0.05,{ }^{* *} \mathrm{P}<0.01$, **** $<0.001$ vs. control. CONT, control; LPS, lipopolysaccharide; HS, heat stress.
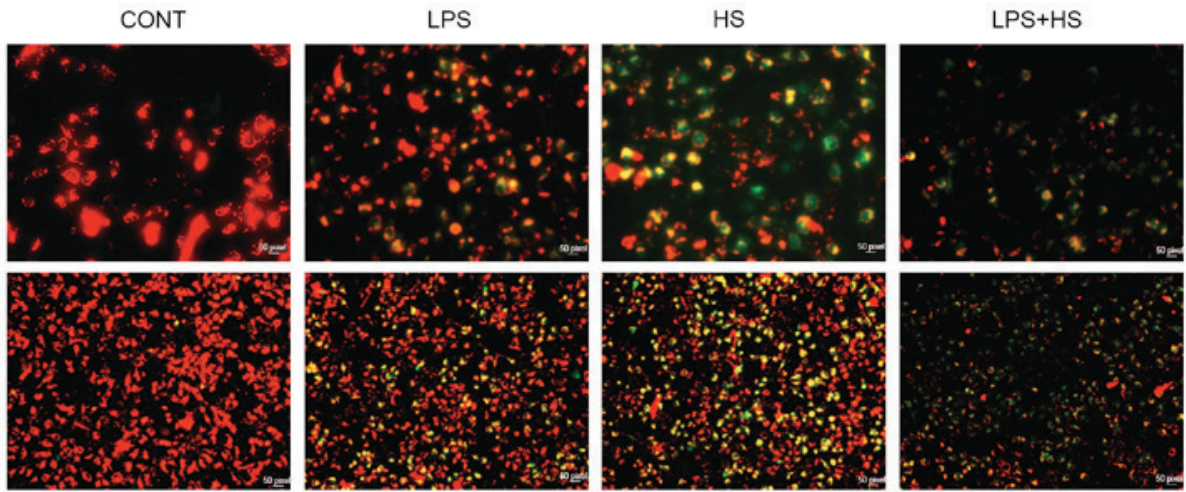

Figure 2. Effects of heat stress and LPS on the mitochondrial membrane potential in IEC-6 cells. IEC- 6 cells were treated with LPS $(1 \mu \mathrm{g} / \mathrm{ml})$, heat stress $\left(42^{\circ} \mathrm{C}\right.$ for $60 \mathrm{~min}$ ) or a combination of LPS and HS. The mitochondrial membrane potential was assayed by 5,5',6,6'-tetrachloro-1,1',3,3'tetraethylbenzimidazo carbocyanine iodide staining. Upper and lower images have magnifications of x 200 and x40, respectively. CONT, control; LPS, lipopolysaccharide; HS, heat stress. 

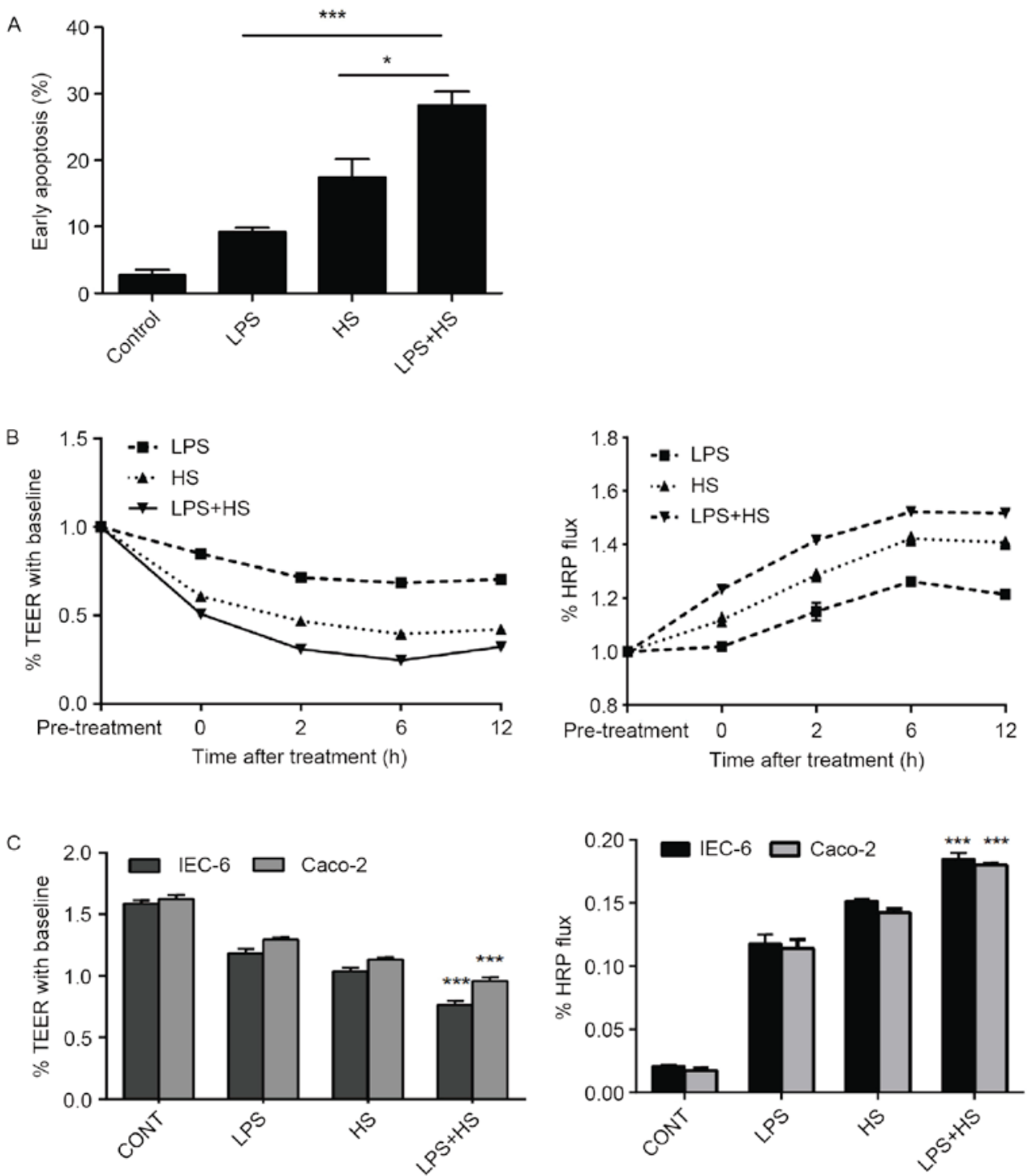

Figure 3. Effects of heat stress, LPS, and a combination of LPS and HS treatment on IEC-6 cell early apoptosis and intestinal epithelial barrier function. IEC-6 cells were treated with LPS $(1 \mu \mathrm{g} / \mathrm{ml})$, HS $\left(42^{\circ} \mathrm{C}\right.$ for $\left.60 \mathrm{~min}\right)$ or a combination of LPS and HS. (A) Quantification of flow cytometry following Annexin V-fluorescein isothiocyanate/propidium iodide staining. ${ }^{*} \mathrm{P}<0.05$ and ${ }^{* * * *} \mathrm{P}<0.001$. TEER and HRP permeability analysis of epithelial barrier function of (B) IEC- 6 cells after 0, 2, 6 and $12 \mathrm{~h}$ treatment, and (C) IEC- 6 and Caco- 2 cells in the different treatment groups. Data are presented as the mean \pm standard deviation of three independent experiments. ${ }^{* * *} \mathrm{P}<0.001$ vs. HS and LPS groups. TEER, transepithelial electrical resistance; HRP, horseradish peroxidase; HS, heat stress; CONT, control; LPS, lipopolysaccharide.

Caco- 2 cells were used as a model to form a typical TJ structure similar to the mature intestinal epithelium in vitro (19). Epithelial barrier integrity and paracellular permeability were determined by the measurement of TEER and flux of HRP. As basal resistance slightly differed in independent wells, the data are presented relative (\% TEER) to baseline (prior to heat exposure $=1$ ). The permeability for HRP into the basolateral chambers, which was determined by the calculated flux, was expressed as a percentage of added HRP marker.

LPS, heat stress and LPS combined with heat stress resulted in a reduction of TEER in time-dependent manner, and a significant increase in paracellular permeability of HRP flux in IEC-6 cells (Fig. 3B). The significant reduction in TEER was accompanied by an increase in paracellular permeability of HRP flux in the LPS combined with heat stress group, compared with the LPS and HS groups at $6 \mathrm{~h}$ after treatment (Fig. 3C). These results indicated that LPS combined with heat stress significantly weakened the intestinal epithelial barrier function, associated with the reduction in TEER and the increase in HRP permeability. In addition, IEC-6 and Caco-2 cells demonstrated the same trend.

Influence of heat stress and LPS on MAPK activation. To determine whether p38, ERK and JNK phosphorylation is required for apoptosis, IEC-6 cells were stimulated with LPS combined with heat stress. As presented in Fig. 4, heat stress alone or LPS combined with heat stress induced activation of p38, ERK and JNK. The phosphorylation levels of p38, ERK and JNK in the LPS combined with heat stress groups were significantly increased compared with the heat stress only groups. However, LPS only slightly increased p38, ERK and JNK phosphorylation levels compared with the control groups. A previous study indicated that LPS induces MAPK phosphorylation at an early time point, which then rapidly decreases to 

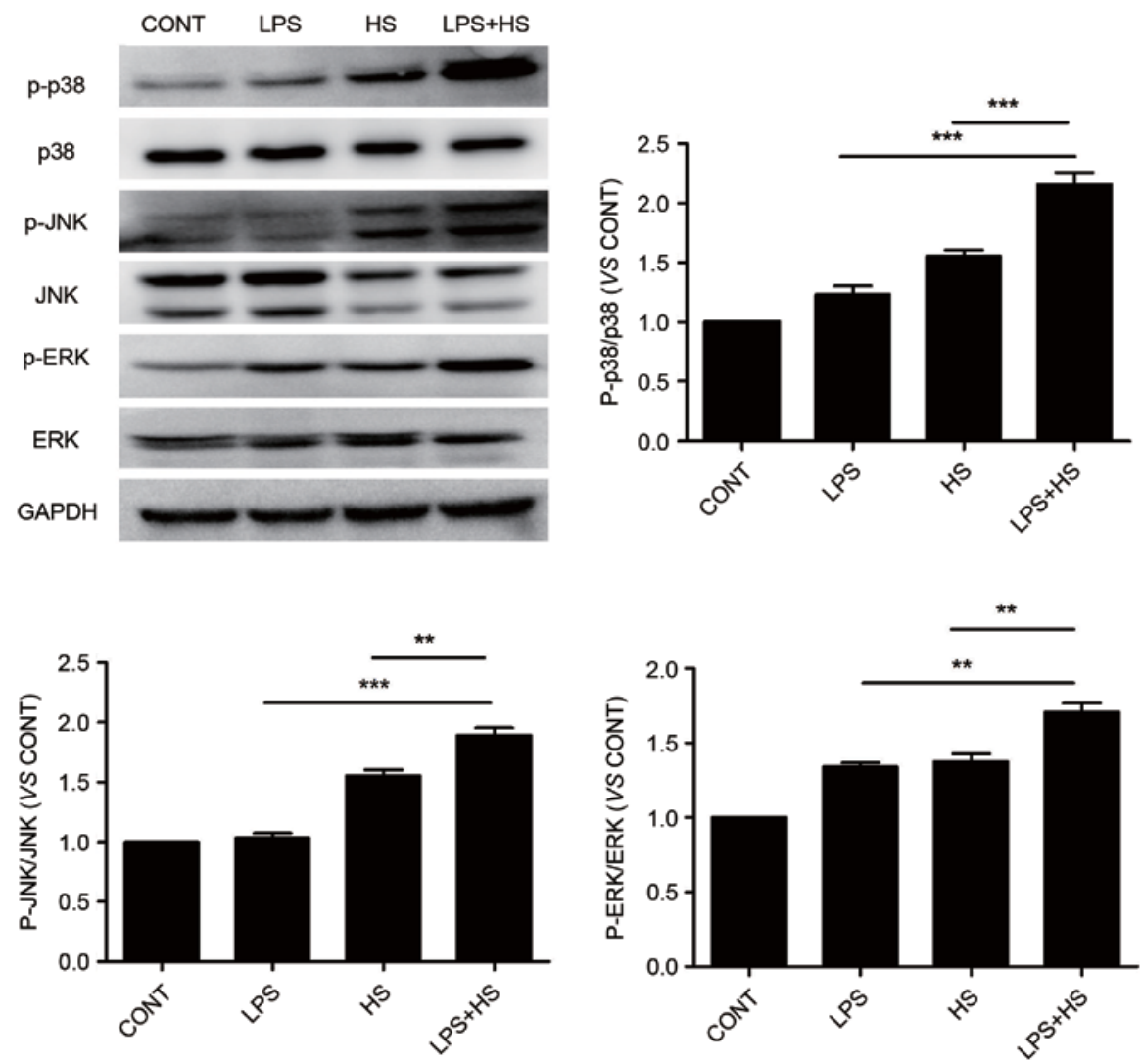

Figure 4. Effects of HS, LPS, or a combination of LPS and HS treatment on p38, ERK and JNK phosphorylation in IEC-6 cells. IEC-6 cells were treated with LPS $(1 \mu \mathrm{g} / \mathrm{ml})$, HS ( $42^{\circ} \mathrm{C}$ for $\left.60 \mathrm{~min}\right)$ or a combination of LPS and HS. Protein expression levels of p-p38, p38, p-JNK and JNK were detected by western blotting. GAPDH served as an internal control. Data are presented as the mean \pm standard deviation of three independent experiments. ${ }^{* *} \mathrm{P}<0.01$ and ${ }^{* * *} \mathrm{P}<0.001$. LPS, lipopolysaccharide; HS, heat stress; JNK, c-Jun N-terminal kinase; ERK, extracellular signal-regulated kinase; p, phosphorylated; CONT, control.
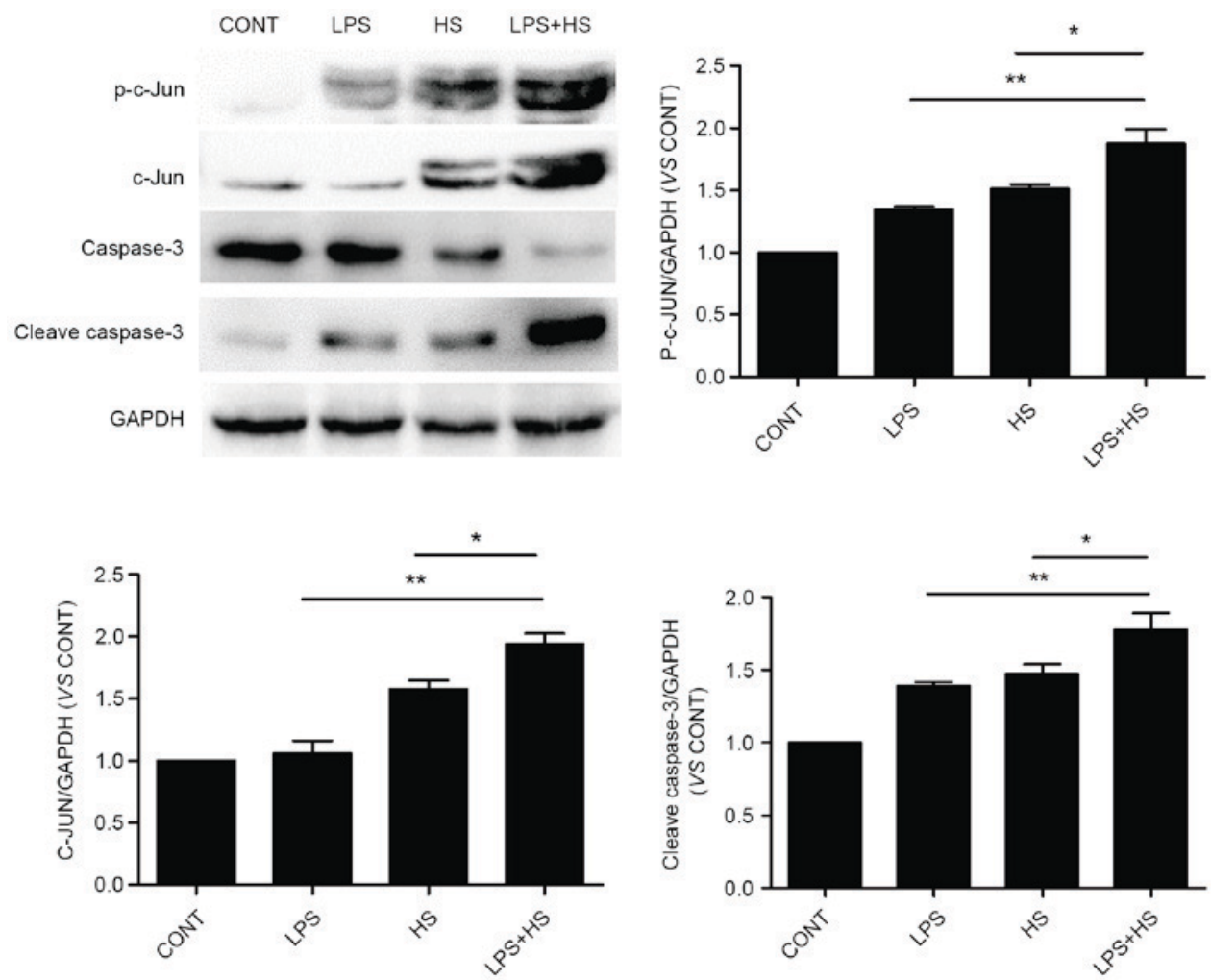

Figure 5. Effects of HS, LPS, or a combination of LPS and HS treatment on c-Jun and caspase-3 activation in IEC-6 cells. IEC-6 cells were treated with LPS $(1 \mu \mathrm{g} / \mathrm{ml})$, HS $\left(42^{\circ} \mathrm{C}\right.$ for $\left.60 \mathrm{~min}\right)$ or a combination of LPS and HS. Protein expression levels of p-c-Jun, c-Jun, caspase- 3 and cleaved caspase- 3 were detected by western blotting. GAPDH served as an internal control. Data are presented as the mean \pm standard deviation of three independent experiments. ${ }^{*} \mathrm{P}<0.05$ and ${ }^{* *} \mathrm{P}<0.01$. LPS, lipopolysaccharide; HS, heat stress; CONT, control; $\mathrm{p}$, phosphorylated. 
A

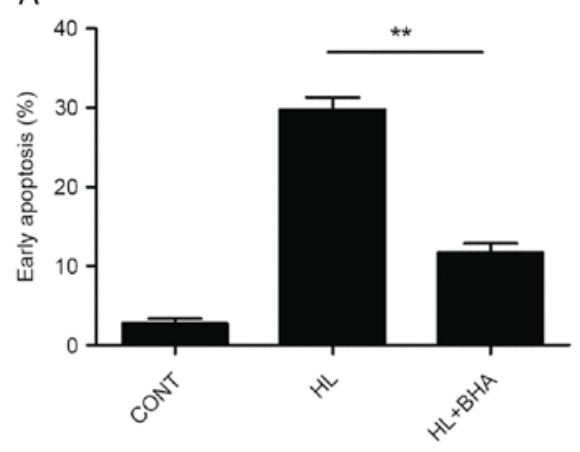

C

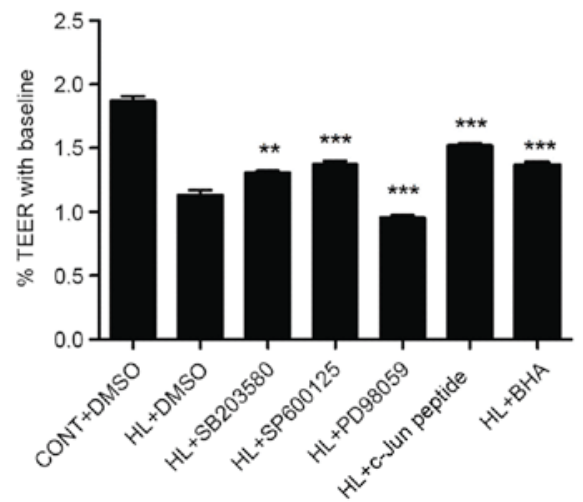

B

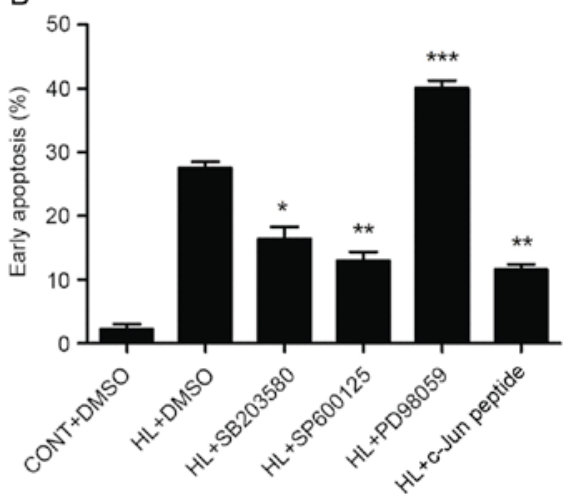

D

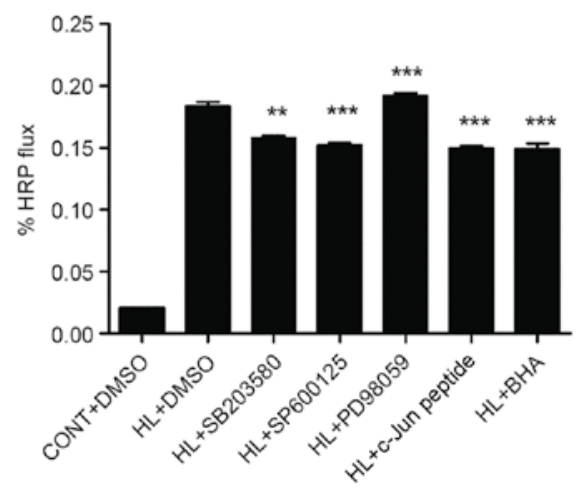

Figure 6. ROS, MAPKs and c-Jun are involved in LPS combined with heat stress-induced IEC-6 cell apoptosis and intestinal epithelial barrier disruption. Early apoptosis rates of cells that were pretreated with (A) BHA and (B) DMSO, SB203580, SP600125, PD98059 or c-Jun peptide, prior to HL treatment followed by a recovery period for $6 \mathrm{~h}$, as assessed by flow cytometry. Intestinal epithelial barrier function analysis following treatment with inhibitors, as detected by (C) TEER and (D) HRP permeability. Data are presented as the mean \pm standard deviation of three independent experiments. ${ }^{* *} \mathrm{P}<0.01$ in $\mathrm{A}$; ${ }^{*} \mathrm{P}<0.05,{ }^{* *} \mathrm{P}<0.01$ and ${ }^{* * *} \mathrm{P}<0.001$ vs. HL + DMSO group. CONT, control; LPS, lipopolysaccharide; HL, combined treatment with LPS and heat stress; DMSO, dimethyl sulfoxide; BHA, butylated hydroxyanisole; TEER, transepithelial electrical resistance; HRP, horseradish peroxidase.

baseline levels, which coincides with ROS generation after a 6-h recovery period from LPS, and induced ROS accumulation was cleared (15). This may explain the low activation level of MAPKs induced by LPS observed in the present study.

Influence of heat stress and LPS on c-Jun and caspase-3 activation. To examine the effects of heat stress and LPS on c-Jun activation and expression, IEC-6 cells were exposed to simultaneous treatment with a combination of LPS $(1 \mu \mathrm{g} / \mathrm{ml})$ and heat stress $\left(42^{\circ} \mathrm{C}\right)$ for $60 \mathrm{~min}$, or LPS and heat stress only, the cells were returned to a temperature of $37^{\circ} \mathrm{C}$ for $6 \mathrm{~h}$. As presented in Fig. 5, heat stress and LPS combined with heat stress caused an increase in the phosphorylation and expression levels of c-Jun. The phosphorylation and expression levels of c-Jun in the LPS combined with heat stress groups was significantly increased compared with the heat stress groups. LPS only slightly increased c-Jun phosphorylation, without affecting its expression. Cleaved caspase-3 expression levels, which typically indicates apoptosis, were detected in the LPS, heat stress and LPS combined with heat stress groups. Cleaved caspase-3 expression in LPS combined with HS groups was significantly increased compared with the heat stress and LPS groups.

Contribution of ROS, MAPKs and c-Jun to LPS combined with heat stress-induced cell apoptosis and intestinal epithelial barrier disruption in IEC-6 cells. To examine whether accumulated ROS levels, or MAPK and c-Jun activation are involved in LPS and heat stress-induced cell apoptosis and intestinal epithelial barrier disruption, IEC-6 cells were stimulated with LPS combined with heat stress in the presence or absence of inhibitors for ROS, MAPKs or c-Jun. As presented in Fig. 6A, treatment with BHA significantly decreased early apoptosis rates. Furthermore, while IEC-6 cells pretreated with PD98059, a specific inhibitor of ERK, did not exhibit an increase in cell survival, cells that were pretreated with specific inhibitors of JNK, p38 and c-Jun (SP600125, SB203580 and c-Jun peptide, respectively) exhibited a significant increase in cell survival (Fig. 6B). Epithelial barrier integrity and paracellular permeability were determined by the measurement of TEER and flux of HRP, respectively. The results of intestinal epithelial barrier integrity and permeability were consistent with apoptosis, as presented in Fig. 6C and D.

ROS mediates activation of MAPKs and c-Jun. As presented in Fig. 7A, LPS combined with heat stress rapidly induced MAPK phosphorylation, which was maintained at high levels for $>60 \mathrm{~min}$. When pretreated with BHA, the phosphorylation level of p38 and ERK were inhibited slightly compared with LPS combined with heat stress without BHA pretreatment. However, the phosphorylation of JNK was not inhibited. To examine the effects of LPS combined with heat stress on c-Jun activation and expression, cells were exposed to heat stress and 
A

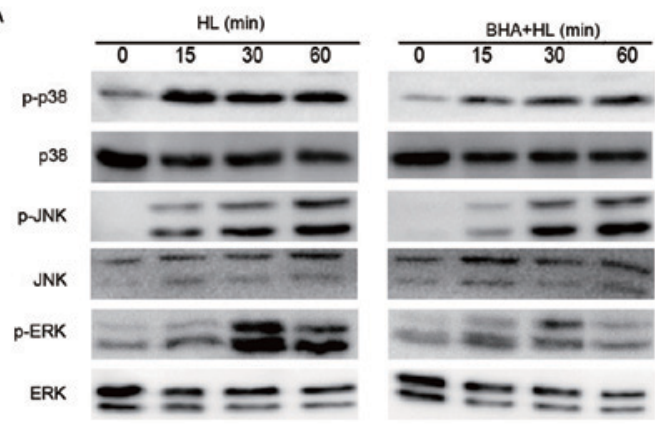

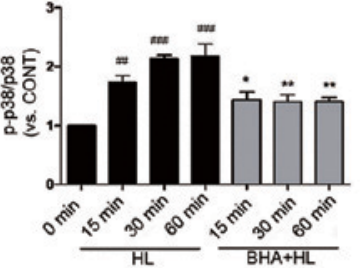
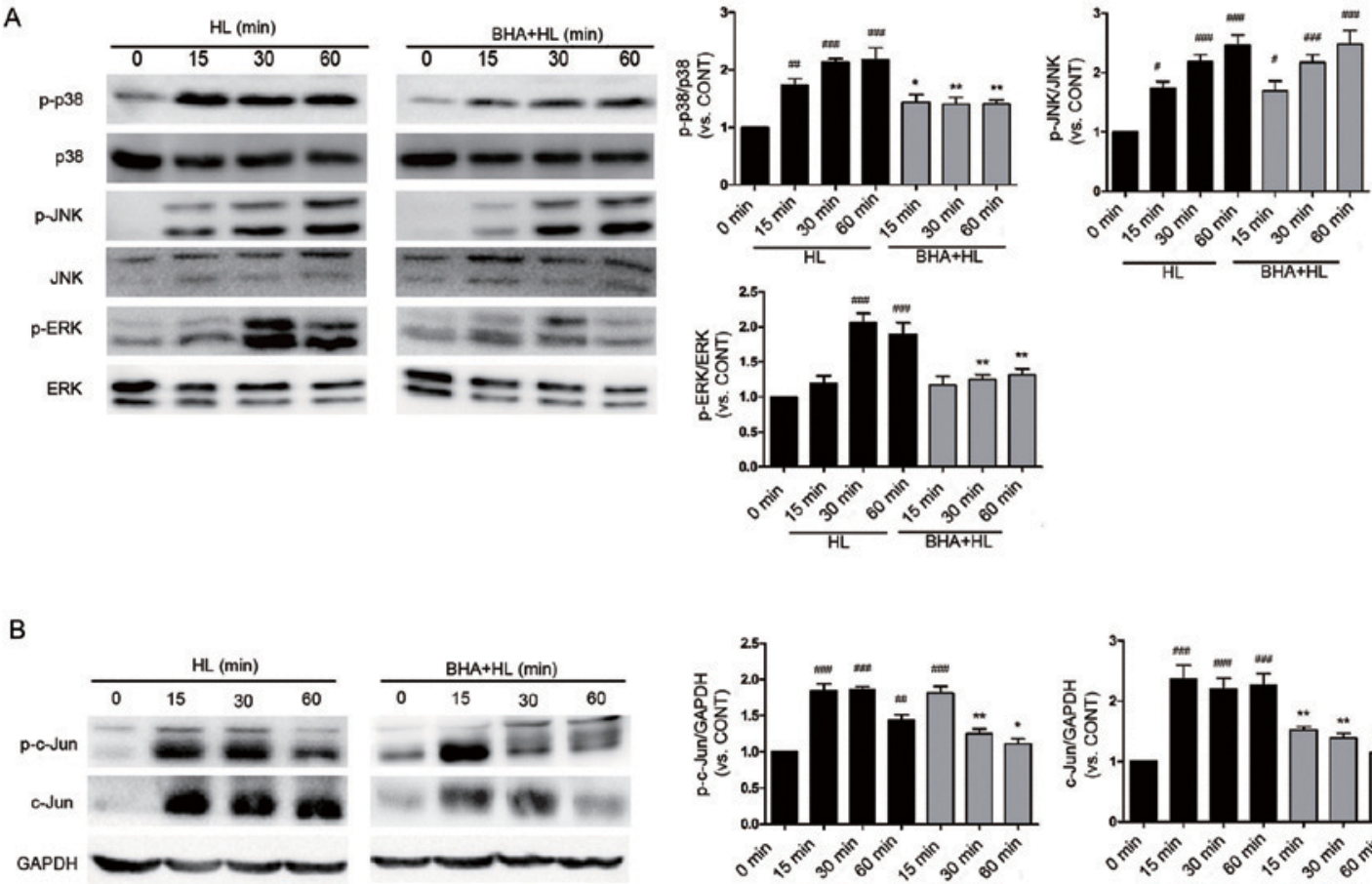

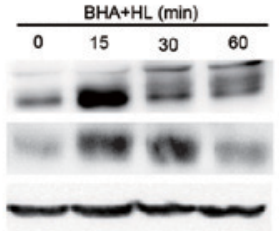

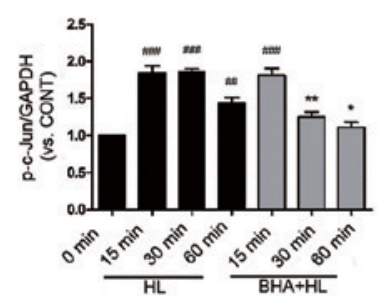

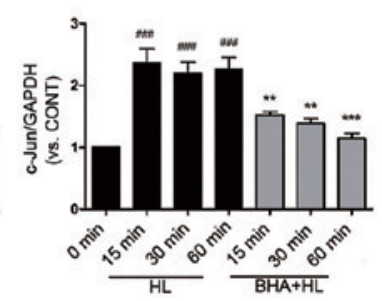

Figure 7. BHA inhibits p38, ERK and c-Jun activation following LPS combined with heat stress treatment in IEC-6 cells. Representative western blot images of protein expression levels of (A) p-p38, p-JNK and p-ERK, and (B) p-c-Jun and c-Jun in IEC-6 cells following pretreatment with BHA prior to HL treatment. Total $\mathrm{p} 38$, total JNK, total ERK and GAPDH served as internal controls. Each band is representative of three experiments. ${ }^{*} \mathrm{P}<0.05$, ${ }^{* * *} \mathrm{P}<0.01$ and ${ }^{* * * *} \mathrm{P}<0.001$ vs. HL group at same time point, ${ }^{\#} \mathrm{P}<0.05,{ }^{\# \#} \mathrm{P}<0.01$ and ${ }^{\# \# \#} \mathrm{P}<0.001$ vs. 0 min group. LPS, lipopolysaccharide; HL, combined treatment with LPS and heat stress; BHA, butylated hydroxyanisole; JNK, c-Jun N-terminal kinase; ERK, extracellular signal-regulated kinase; p, phosphorylated; CONT, 0 min control group.

LPS for $0,15,30$ or 60 min. LPS combined with heat stress led to rapid induction of c-Jun phosphorylation on serine 63 and subsequent accumulation of c-Jun protein in time-dependent manner. When pretreated with BHA, c-Jun phosphorylation and expression was inhibited within $60 \mathrm{~min}$ (Fig. 7B). These results demonstrated that $\mathrm{p} 38$, ERK and c-Jun activation are downstream events of ROS accumulation.

\section{Discussion}

It has previously been reported that endotoxin levels in the blood increase in heatstroke patients at a mean rectal temperature of $42 \cdot 1^{\circ} \mathrm{C}(19,20)$. Intestinal permeability to endotoxin or LPS from the gut entering the circulation increases in heat-stressed animals $(4,21)$. On the contrary, anti-LPS antibodies protect against the transition from heat stress to heatstroke (1). This suggests that LPS and heat stress may serve an important role in the pathogenesis of heatstroke. The present study mimicked the micro-environment of intestinal epithelial cells under severe heat stress conditions to investigate cell apoptosis and its potential underlying mechanisms.

In the present study, LPS and heat stress induced production of ROS, mitochondrial membrane potential disruption and cell apoptosis which eventually led to increased intestinal permeability and reduced epithelial resistance in IEC-6 cells. Furthermore, ROS production contributed to activation of $\mathrm{p} 38$, ERK and c-Jun. In previous studies, intracellular ROS production was increased by heat stress in endothelial cells (6), and IEC-6 cells by LPS in macrophages (5). Previous studies have suggested that environmental heat stress may stimulate production of ROS, which contributes to intestinal barrier dysfunction and cell apoptosis (22). As predicted, heat stress or LPS stimulation induced early apoptosis in IEC-6 cells, a synergistic effect produced when heat stress and LPS stimulation were combined. A similar profile was obtained when the corresponding cell lysates were analyzed for cleaved caspase-3 and activation of MAPKs and c-Jun. These results indicated that the heat stroke environment may induce multiple types of cell damage mediated by different molecules and signaling pathways.

Studies have demonstrated that the level of ROS is associated with cell apoptosis. The present study revealed that cell apoptosis may be inhibited by using the ROS scavenger, BHA, which is consistent with a previous study (23). Cell apoptosis was assessed using the inhibitors of JNK, p38, ERK and c-Jun by flow cytometry. Activation of JNK, p38 and c-Jun serve pro-apoptotic roles, whereas ERK is resistant to apoptosis. It is hypothesized that ERK is important for cell survival, while JNK and p38 have been characterized as stress-responsive factors, thus serving important roles in apoptosis (12-16). In addition, the phosphorylation of c-Jun is required for apoptosis following survival signal withdrawal (24-27). These conclusions were obtained in the present study. Therefore, the combination of LPS and heat stress may induce both pro- and anti-apoptotic signaling pathways via a ROS-MAPK/c-Jun signaling pathway in IEC- 6 cells.

Previous studies have demonstrated that ROS mediates MAPK and c-Jun activation in various cellular stress conditions and cell types $(4,26,28)$. To investigate the molecular mechanism underlying MAPK and c-Jun activation, cells were 
pretreated with an antioxidant. The results indicated that the phosphorylation levels of p38, ERK and c-Jun are suppressed. However, JNK phosphorylation levels did not alter significantly. These results demonstrated that p38, ERK and c-Jun activation is a downstream event of ROS accumulation.

In conclusion, the results reported in the present study suggested that combined LPS and heat stress contributed to IEC- 6 cell apoptosis and intestinal epithelial barrier disruption, and that more than one single mechanism was involved. The present study demonstrated a critical role of ROS modulating LPS combined with heat stress-induced MAPK and c-Jun activation in IEC- 6 cells. Elucidation of the mechanism by which ROS regulates activation of MAPKs and c-Jun may facilitate understanding of pathological conditions involving ROS, such as heat stroke.

\section{Acknowledgements}

The present study was supported by the National Natural Science Foundation of China (grant no. 81471839) and the project team of the Natural Science Foundation of Guangdong Province (grant no. s2013030013217).

\section{References}

1. Wang X, Yuan B, Dong W, Yang B, Yang Y, Lin X and Gong G: Humid heat exposure induced oxidative stress and apoptosis in cardiomyocytes through the angiotensin II signaling pathway, Heart Vessels 30: 396-405, 2015.

2. Gathiram P, Wells MT, Brock-Utne JG and Gaffin SL: Antilipopolysaccharide improves survival in primates subjected to heat stroke. Circ Shock 23: 157-164, 1987.

3. Yang PC, He SH and Zheng PY: Investigation into the signal transduction pathway via which heat stress impairs intestinal epithelial barrier function. J Gastroenterol Hepatol 22: 1823-1831, 2007.

4. Gathiram P, Wells MT, Raidoo D, Brock-Utne JG and Gaffin SL: Portal and systemic plasma lipopolysaccharide concentrations in heat-stressed primates. Circ Shock 25: 223-230, 1988.

5. Xiao G, Tang L, Yuan F, Zhu W, Zhang S, Liu Z, Geng Y, Qiu X, Zhang Y and Su L: Eicosapentaenoic acid enhances heat stress-impaired intestinal epithelial barrier function in Caco-2 cells. PLoS One 8: e73571, 2013.

6. Gu ZT, Wang H, Li L, Liu YS, Deng XB, Huo SF, Yuan FF, Liu ZF, Tong HS and Su L: Heat stress induces apoptosis through transcription-independent p53-mediated mitochondrial pathways in human umbilical vein endothelial cell. Sci Rep 4: 4469, 2014.

7. Yokochi T, Morikawa A, Kato Y, Sugiyama T and Koide N: Apoptotic cell death in response to LPS. Prog Clin Biol Res 397: 235-242, 1998.

8. Indo HP, Yen HC, Nakanishi I, Matsumoto K, Tamura M, Nagano Y, Matsui H, Gusev O, Cornette R, Okuda T, et al: A mitochondrial superoxide theory for oxidative stress diseases and aging. J Clin Biochem Nutr 56: 1-7, 2015.

9. Fiers W, Beyaert R, Declercq W and Vandenabeele P: More than one way to die: Apoptosis, necrosis and reactive oxygen damage. Oncogene 18: 7719-7730, 1999

10. Lee JA, Song HY, Ju SM, Lee SJ, Kwon HJ, Eum WS, Jang SH, Choi SY and Park JS: Differential regulation of inducible nitric oxide synthase and cyclooxygenase-2 expression by superoxide dismutase in lipopolysaccharide stimulated RAW 264.7 cells. Exp Mol Med 41: 629-637, 2009.
11. Skibba JL, Powers RH, Stadnicka A, Cullinane DW, Almagro UA and Kalbfleisch JH: Oxidative stress as a precursor to the irreversible hepatocellular injury caused by hyperthermia. Int J Hyperthermia 7: 749-761, 1991

12. Burdon RH, Gill VM and Rice-Evans C: Oxidative stress and heat shock protein induction in human cells. Free Radic Res Commun 3: 129-139, 1987.

13. Kim EK and Choi EJ: Compromised MAPK signaling in human diseases: An update. Arch Toxicol 89: 867-882, 2015.

14. Lee SI, Min KS, Bae WJ, Lee YM, Lee SY, Lee ES and Kim EC: Role of SIRT1 in heat stress- and lipopolysaccharide-induced immune and defense gene expression in human dental pulp cells. J Endod 37: 1525-1530, 2011.

15. Sakon S, Xue X, Takekawa M, Sasazuki T, Okazaki T, Kojima Y, Piao JH, Yagita H, Okumura K, Doi T and Nakano H: NF-kappaB inhibits TNF-induced accumulation of ROS that mediate prolonged MAPK activation and necrotic cell death. EMBO J 22: 3898-3909, 2003.

16. Guo X, Chen S, Zhang Z, Dobrovolsky VN, Dial SL, Guo L and Mei N: Reactive oxygen species and c-Jun N-terminal kinases contribute to TEMPO-induced apoptosis in L5178Y cells. Chem Biol Interact 235: 27-36, 2015.

17. Bai L, Mao R, Wang J, Ding L, Jiang S, Gao C, Kang H, Chen X, Sun $\mathrm{X}$ and $\mathrm{Xu}$ J: ERK1/2 promoted proliferation and inhibited apoptosis of human cervical cancer cells and regulated the expression of c-Fos and c-Jun proteins. Med Oncol 32: 57, 2015.

18. Su SH, Wu YF, Lin Q, Yu F and Hai J: Cannabinoid receptor agonist WIN55,212-2 and fatty acid amide hydrolase inhibitor URB597 suppress chronic cerebral hypoperfusion-induced neuronal apoptosis by inhibiting c-Jun N-terminal kinase signaling. Neuroscience 301: 563-575, 2015.

19. Sambuy Y, De Angelis I, Ranaldi G, Scarino ML, Stammati A and Zucco F: The Caco-2 cell line as a model of the intestinal barrier: Influence of cell and culture-related factors on Caco-2 cell functional characteristics. Cell Biol Toxicol 21: 1-26, 2005.

20. Dörfel MJ and Huber O: Modulation of tight junction structure and function by kinases and phosphatases targeting occludin, J Biomed Biotechnol 2012: 807356, 2012.

21. Kimura Y, Shiozaki H, Hirao M, Maeno Y, Doki Y, Inoue M, Monden T, Ando-Akatsuka Y, Furuse M, Tsukita S and Monden M: Expression of occludin, tight-junction-associated protein, in human digestive tract. Am J Pathol 151: 45-54, 1997.

22. Shapiro Y, Alkan M, Epstein Y, Newman F and Magazanik A: Increase in rat intestinal permeability to endotoxin during hyperthermia. Eur J Appl Physiol Occup Physiol 55: 410-412, 1986.

23. Hall DM, Buettner GR, Oberley LW, Xu L, Matthes RD and Gisolfi CV: Mechanisms of circulatory and intestinal barrier dysfunction during whole body hyperthermia. Am J Physiol Heart Circ Physiol 280: H509-H521, 2001.

24. Liu L, Fu J, Li T, Cui R, Ling J, Yu X, Ji H and Zhang Y: NG, a novel PABA/NO-based oleanolic acid derivative, induces human hepatoma cell apoptosis via a ROS/MAPK-dependent mitochondrial pathway. Eur J Pharmacol 691: 61-68, 2012.

25. Zhu J, Zhang J, Huang H, Li J, Yu Y, Jin H, Li Y, Deng X, Gao J, Zhao Q and Huang C: Crucial Role of c-Jun phosphorylation at Ser63/73 mediated by PHLPP protein degradation in the cheliensisin a inhibition of cell transformation. Cancer Prev Res (Phila) 7: 1270-1281, 2014.

26. Herdman ML, Marcelo A, Huang Y, Niles RM, Dhar S and Kiningham KK: Thimerosal induces apoptosis in a neuroblastoma model via the cJun N-terminal kinase pathway. Toxicol Sci 92: 246-253, 2006.

27. Watson A, Eilers A, Lallemand D, Kyriakis J, Rubin LL and Ham J: Phosphorylation of c-Jun is necessary for apoptosis induced by survival signal withdrawal in cerebellar granule neurons. J Neurosci 18: 751-762, 1998.

28. Qin Z, Robichaud P, He T, Fisher GJ, Voorhees JJ and Quan T: Oxidant exposure induces cysteine-rich protein 61 (CCN1) via c-Jun/AP-1 to reduce collagen expression in human dermal fibroblasts. PLoS One 9: e115402, 2014. 